\title{
New Quasi-Exactly Solvable Sextic Polynomial Potentials
}

\author{
Carl M. Bender* and Maria Monou \\ Blackett Laboratory, Imperial College, London SW7 2BZ, UK
}

(Dated: November 13, 2018)

\begin{abstract}
A Hamiltonian is said to be quasi-exactly solvable (QES) if some of the energy levels and the corresponding eigenfunctions can be calculated exactly and in closed form. An entirely new class of QES Hamiltonians having sextic polynomial potentials is constructed. These new Hamiltonians are different from the sextic QES Hamiltonians in the literature because their eigenfunctions obey $\mathcal{P} \mathcal{T}$-symmetric rather than Hermitian boundary conditions. These new Hamiltonians present a novel problem that is not encountered when the Hamiltonian is Hermitian: It is necessary to distinguish between the parametric region of unbroken $\mathcal{P} \mathcal{T}$ symmetry, in which all of the eigenvalues are real, and the region of broken $\mathcal{P} \mathcal{T}$ symmetry, in which some of the eigenvalues are complex. The precise location of the boundary between these two regions is determined numerically using extrapolation techniques and analytically using WKB analysis.
\end{abstract}

PACS numbers: 03.65.Sq, 02.70.Hm, 02.90.+p

\section{SEXTIC QES HAMILTONIANS}

The purpose of this paper is to introduce a new class of quasi-exactly solvable (QES) Hamiltonians having sextic polynomial potentials. While these new kinds of QES Hamiltonians have positive, real eigenvalues, they have not yet been discussed in the literature because they are not Hermitian. Instead, they are $\mathcal{P} \mathcal{T}$ symmetric.

The term quasi-exactly solvable (QES) is used to describe a quantum-mechanical Hamiltonian when a finite portion of its energy spectrum and associated eigenfunctions can be found exactly and in closed form [1]. Typically, QES potentials depend on a parameter $J$, and for positive integer values of $J$ one can find exactly the first $J$ eigenvalues and eigenfunctions, usually of a given parity. It has been shown that QES systems can be classified by using an algebraic approach in which the Hamiltonian is expressed in terms of the generators of a Lie algebra [2, 3, 4, 5].

Perhaps the simplest example of a QES Hamiltonian having a sextic potential is [6, 7]

$$
H=p^{2}+x^{6}-(4 J-1) x^{2},
$$

where $J$ is a positive integer. For each positive integer value of $J$, the time-independent Schrödinger equation for this Hamiltonian,

$$
-\psi^{\prime \prime}(x)+\left[x^{6}-(4 J-1) x^{2}\right] \psi(x)=E \psi(x),
$$

has $J$ even-parity eigenfunctions in the form of an exponential times a polynomial:

$$
\psi(x)=e^{-x^{4} / 4} \sum_{k=0}^{J-1} c_{k} x^{2 k} .
$$

\footnotetext{
* Permanent address: Department of Physics, Washington University, St. Louis, MO 63130, USA.
} 
The polynomial coefficients $c_{k}(0 \leq k \leq J-1)$ satisfy the recursion relation

$$
4(J-k) c_{k-1}+E c_{k}+2(k+1)(2 k+1) c_{k+1}=0,
$$

where we define $c_{-1}=c_{J}=0$. The simultaneous linear equations (4) have a nontrivial solution for $c_{0}, c_{1}, \ldots, c_{J-1}$ if the determinant of the coefficients vanishes. This determinant is a polynomial of degree $J$ in the variable $E$. The roots of this polynomial are all real and are the $J$ quasi-exact energy eigenvalues of the Hamiltonian (11). Note that all of the QES eigenfunctions (3) of $H$ in (11) have the form of a decaying exponential $\exp \left(-\frac{1}{4} x^{4}\right)$ multiplying a polynomial. This is the standard form in the literature for the eigenfunctions of any QES Hamiltonian whose potential is a polynomial.

The QES Hamiltonians associated with Hermitian Hamiltonians have been examined in depth and classified exhaustively [1]. However, in 1998 new kinds of Hamiltonians that have positive real energy levels were discovered [8, 9]. These new kinds of Hamiltonians are not Hermitian $\left(H \neq H^{\dagger}\right)$ in the usual Dirac sense, where the Dirac adjoint symbol $\dagger$ represents combined transpose and complex conjugation. Instead, these Hamiltonians possess $\mathcal{P} \mathcal{T}$ symmetry $H=H^{\mathcal{P T}}$; that is, they remain invariant under combined space and time reflection. This new class of non-Hermitian Hamiltonians has been studied heavily 10 , 11, 12] and it has been shown that when the $\mathcal{P} \mathcal{T}$ symmetry is not broken, such Hamiltonians define unitary theories of quantum mechanics [13.

The key difference between Hermitian Hamiltonians and complex, non-Hermitian, $\mathcal{P} \mathcal{T}$ symmetric Hamiltonians is that with $\mathcal{P} \mathcal{T}$-symmetric Hamiltonians the boundary conditions on the eigenfunctions (the solutions to the time-independent Schrödinger equation) are imposed in wedges in the complex plane. Sometimes these wedges do not include the real axis. (A detailed discussion of the complex asymptotic behavior of solutions to eigenvalue problems may be found in Ref. [14].)

The discovery of $\mathcal{P} \mathcal{T}$-symmetric Hamiltonians was followed immediately by the discovery of a new class of QES models. Until 1998 it was thought that if the potential associated with a QES Hamiltonian was a polynomial, then this polynomial had to be at least sextic; its degree could not be less than six. This property is in fact true for Hamiltonians that are Hermitian. However, in 1998 it was discovered that it is possible to have a QES nonHermitian complex Hamiltonian whose potential is quartic [15]:

$$
H=p^{2}-x^{4}+2 i a x^{3}+\left(a^{2}-2 b\right) x^{2}+2 i(a b-J) x .
$$

Here, $a$ and $b$ are real parameters and $J$ is a positive integer. For a large region of the parameters $a$ and $b$, the energy levels of this family of quartic Hamiltonians are real, discrete, and bounded below, and the quasi-exact portion of the spectra consists of the lowest $J$ eigenvalues. Like the eigenvalues of the Hamiltonian (11), the lowest $J$ eigenvalues of these potentials are the roots of a $J$ th-degree polynomial [16].

The reality of the eigenvalues of $H$ in (15) is ensured by the boundary conditions that its eigenfunctions are required to satisfy. The eigenfunctions are required to vanish as $|x| \rightarrow \infty$ in the complex- $x$ plane inside of two wedges called Stokes wedges. The right wedge is bounded above and below by lines at $0^{\circ}$ and $-60^{\circ}$ and the left wedge is bounded above and below by lines at $-180^{\circ}$ and $-120^{\circ}$. The leading asymptotic behavior of the wave function inside these wedges is given by

$$
\psi(x) \sim e^{-i x^{3} / 3} \quad(|x| \rightarrow \infty) .
$$


The new class of QES sextic Hamiltonians reported in this paper has the form

$$
H=p^{2}+x^{6}+2 a x^{4}+\left(4 J-1+a^{2}\right) x^{2},
$$

where $J$ is a positive integer and $a$ is a real parameter. These Hamiltonians are very similar in structure to those in (11) and to the other QES sextic Hamiltonians discussed in the literature [1], but their distinguishing characteristic is that the asymptotic behavior of their eigenfunctions in the complex- $x$ plane is different.

Let us examine first the asymptotic behavior of the eigenfunction solutions to the Schrödinger equation (21). For brevity, we call the eigenfunctions in (3) the good solutions to (2) because they satisfy the physical requirement of being quadratically integrable. These good solutions decay exponentially like $\exp \left(-\frac{1}{4} x^{4}\right)$ as $x \rightarrow \pm \infty$, while the corresponding linearly independent $b a d$ solutions grow exponentially like $\exp \left(\frac{1}{4} x^{4}\right)$ as $x \rightarrow \pm \infty$. In the complex- $x$ plane the good solutions (3) decay exponentially as $|x| \rightarrow \infty$ in two Stokes wedges that are centered about the positive and the negative real- $x$ axes. These wedges have an angular opening of $45^{\circ}$. The bad solutions grow exponentially in these wedges. At the upper and lower edges of these wedges the good and bad solutions cease to decay and to grow exponentially and they become purely oscillatory.

As we move downward past the lower edges of these wedges, we enter a new pair of Stokes wedges. These wedges also have a $45^{\circ}$ angular opening and are centered about the lines $\arg x=-45^{\circ}$ and $\arg x=-135^{\circ}$. In these lower wedges, the good solutions grow exponentially as $|x| \rightarrow \infty$ and thus they behave like a bad solutions.

In the lower pair of wedges we can find solutions to the new class of Hamiltonians in (7) that behave like good solutions. These new $\mathcal{P} \mathcal{T}$-symmetric eigenfunctions have the general form of the exponential $\exp \left(\frac{1}{4} x^{4}+\frac{1}{2} a x^{2}\right)$ multiplied by a polynomial [17]:

$$
\psi(x)=e^{x^{4} / 4+a x^{2} / 2} \sum_{k=0}^{J-1} c_{k} x^{2 k} .
$$

Hamiltonians having even sextic polynomial potentials are special because such Hamiltonians can be either Hermitian or $\mathcal{P} \mathcal{T}$-symmetric depending on whether the eigenfunctions are required to vanish exponentially in the $45^{\circ}$ wedges containing the positive and negative real- $x$ axes or in the other pair of $45^{\circ}$ wedges contiguous to and lying just below these wedges in the complex- $x$ plane. The solutions for these two different boundary conditions are somewhat related. Specifically, a good solution in one pair of wedges becomes a bad solution in the other pair of wedges. However, a bad solution in one pair of wedges does not become a good solution in the other pair of wedges, as we now explain.

Given a good solution $\psi_{\text {good }}(x)$ in one pair of wedges, we use the method of reduction of order [18] to find the bad solution. We seek a bad solution in the form $\psi_{\text {bad }}(x)=$ $\psi_{\text {good }}(x) u(x)$, where $u(x)$ is an unknown function to be determined. Substituting the bad solution into the Schrödinger equation $-\psi^{\prime \prime}(x)+V(x) \psi(x)=E \psi(x)$, we get the differential equation satisfied by $u(x)$ :

$$
\psi_{\text {good }}(x) u^{\prime \prime}(x)+2 \psi_{\text {good }}^{\prime}(x) u^{\prime}(x)=0 .
$$

We solve this equation by multiplying by the integrating factor $\psi_{\text {good }}(x)$ and obtain the result

$$
\psi_{\text {bad }}(x)=\psi_{\text {good }}(x)\left(\int^{x} d s\left[\psi_{\text {good }}(s)\right]^{-2}+C\right),
$$


where $C$ is an arbitrary constant.

This bad solution always grows exponentially in the two wedges in which the good solution decays exponentially. How does this bad solution behave in the other pair of wedges in which the good solution grows exponentially? We can always choose the constant $C$ so that the bad solution vanishes as $|x| \rightarrow \infty$ in one of these two wedges. However, in the other of the two wedges, the bad solution will always grow exponentially. Thus, while the good solution becomes bad as we cross from one pair of wedges to the other, the bad solution does not become good.

\section{DETERMINATION OF THE $\mathcal{P} \mathcal{T}$ BOUNDARY}

The difference between the Hermitian Hamiltonians in (1) and the $\mathcal{P} \mathcal{T}$-symmetric Hamiltonians in (17) is that the Hermitian Hamiltonians always have real eigenvalues. The $\mathcal{P} \mathcal{T}$ symmetric Hamiltonians in (17) have real eigenvalues only if the $\mathcal{P} \mathcal{T}$ symmetry is unbroken; if the $\mathcal{P} \mathcal{T}$ symmetry is broken, some of the eigenvalues will be complex. Thus, it is crucial to determine whether the $\mathcal{P} \mathcal{T}$ symmetry is broken. We will see that there is a range of values of the parameter $a$ in (77) for which the energy levels are real and this is the region of unbroken $\mathcal{P} \mathcal{T}$ symmetry. Outside of this region some of the eigenvalues appear as complex-conjugate pairs.

Let us illustrate the difference between the regions of broken and unbroken $\mathcal{P} \mathcal{T}$ symmetry by examining some special solutions of the Schrödinger equation

$$
-\psi^{\prime \prime}(x)+\left[x^{6}+2 a x^{4}+\left(4 J-1+a^{2}\right) x^{2}\right] \psi(x)=E \psi(x),
$$

corresponding to $H$ in (77). First, consider the case $J=1$. The unique eigenfunction solution to (11) of the form in (18) is $\psi(x)=\exp \left(\frac{1}{4} x^{4}+\frac{1}{2} a x^{2}\right)$ and the corresponding eigenfunction is $E=-a$. Note that $E$ is real so long as $a$ is real. Thus, for $J=1$ there is no region of broken $\mathcal{P} \mathcal{T}$ symmetry.

Next, consider the case $J=2$. Now, there are two eigenfunctions. The two eigenvalues are given by

$$
E=-3 a \pm 2 \sqrt{a^{2}-2}
$$

Thus, there is now an obvious transition between real eigenvalues (unbroken $\mathcal{P} \mathcal{T}$ symmetry) and complex eigenvalues (broken $\mathcal{P} \mathcal{T}$ symmetry). Evidently, the eigenvalues are real if $a \geq \sqrt{2}$ or if $a \leq-\sqrt{2}$.

We find that for any positive integer value of $J>1$, the eigenvalues $E$ for $H$ in (77) are entirely real if $a^{2}$ is greater than some critical value $\left[a_{\text {crit }}(J)\right]^{2}$ that depends on $J$. These critical values up to $J=20$ are shown in Table 1

Observe from Table \that the critical values of $\left[a_{\text {crit }}\right]^{2}$ grow monotonically with increasing $J$. We have therefore also calculated the differences between successive critical values of $a^{2}$. These differences also grow monotonically with increasing $J$, but they appear to be leveling off and seem to be approaching a limiting value. To see whether the differences are indeed approaching a limiting value as $J$ increases, we have plotted in Fig. 1 these differences as a function of $1 / J$. This plot suggests that the differences tend to the value 12 as $J \rightarrow \infty$.

To determine whether it is true that these differences really do approach limit 12 , it is necessary to extrapolate the sequence of differences to its value at $J=\infty$. To do so we have calculated the Richardson extrapolants [18] of the sequence of differences. The first Richardson extrapolants, $R_{1}(J)$, of these differences are listed in Table II. Observe that 


\begin{tabular}{c|c|c}
$J$ & {$\left[a_{\text {crit }}(J)\right]^{2}$} & {$\left[a_{\text {crit }}(J+1)\right]^{2}-\left[a_{\text {crit }}(J)\right]^{2}$} \\
\hline 2 & 2 & 8.5874700363 \\
3 & 10.5874700363 & 9.9640634033 \\
4 & 20.5515334397 & 10.5019218257 \\
5 & 31.0534552654 & 10.7985017073 \\
6 & 41.8519569727 & 10.9889820601 \\
7 & 52.8409390328 & 11.1226958611 \\
8 & 63.9636348939 & 11.2222406710 \\
9 & 75.1858755649 & 11.2995196186 \\
10 & 86.4853951835 & 11.3614120451 \\
11 & 97.8468072286 & 11.4122263065 \\
12 & 109.2590335351 & 11.4547578245 \\
13 & 120.7137913596 & 11.4909345548 \\
14 & 132.2047259144 & 11.5221201923 \\
15 & 143.7268461067 & 11.5493064512 \\
16 & 155.2761720922 & 11.5732299524 \\
17 & 166.8494020446 & 11.5945096795 \\
18 & 178.4439117241 & 11.6134962251 \\
19 & 190.0574079492 & 11.6306193103 \\
20 & 201.6880273595 & \\
& &
\end{tabular}

TABLE I: Critical values, $\left[a_{\text {crit }}(J)\right]^{2}$, of the parameter $a^{2}$ listed as a function of $J$. When $a^{2}$ is greater than this critical value, the eigenvalues of the $\mathcal{P} \mathcal{T}$-symmetric Hamiltonian $H$ in (7) are all real. Thus, this is the region of unbroken $\mathcal{P} \mathcal{T}$ symmetry. The $\mathcal{P} \mathcal{T}$ symmetry is broken when $a^{2}<\left[a_{\text {crit }}(J)\right]^{2}$. Note that the differences between successive values of $\left[a_{\text {crit }}(J)\right]^{2}$ appear to be approaching a limit and this is indeed the case. In fact, the numerical value of this limit is exactly 12. Thus, for large $J$ the critical values have the simple asymptotic behavior $\left[a_{\text {crit }}(J)\right]^{2} \sim 12 J$.

the sequence $R_{1}(J)$ rises more slowly and quite convincingly appears to be approaching the value 12 . The differences $R_{1}(J+1)-R_{1}(J)$ between successive Richardson extrapolants are also shown.

To test further the hypothesis that $R_{1}(J)$ tends to the limiting value 12 as $J \rightarrow \infty$, we have calculated successive Richardson extrapolants of the Richardson extrapolants $R_{1}(J)$ in Table II. The successive extrapolants are listed in Table III and they provide very strong numerical evidence that $\lim _{J \rightarrow \infty}\left(\left[a_{\text {crit }}(J+1)\right]^{2}-\left[a_{\text {crit }}(J)\right]^{2}\right)=12$. From this we conclude that for large $J$ the asymptotic behavior of the critical value of $a^{2}$ is given by

$$
\left[a_{\text {crit }}(J)\right]^{2} \sim 12 J \quad(J \rightarrow \infty) .
$$

Our numerical analysis provides convincing evidence that for large $J$ the boundary between the regions of broken and unbroken $\mathcal{P} \mathcal{T}$ symmetry is given by the asymptotic behavior in (13). We will now verify this result analytically by using WKB methods [18]. From our numerical analysis we know that the first eigenvalues to become complex conjugate pairs are always the highest, and this implies that WKB is the appropriate tool for investigating the $\mathcal{P} \mathcal{T}$ boundary for large $J$. 


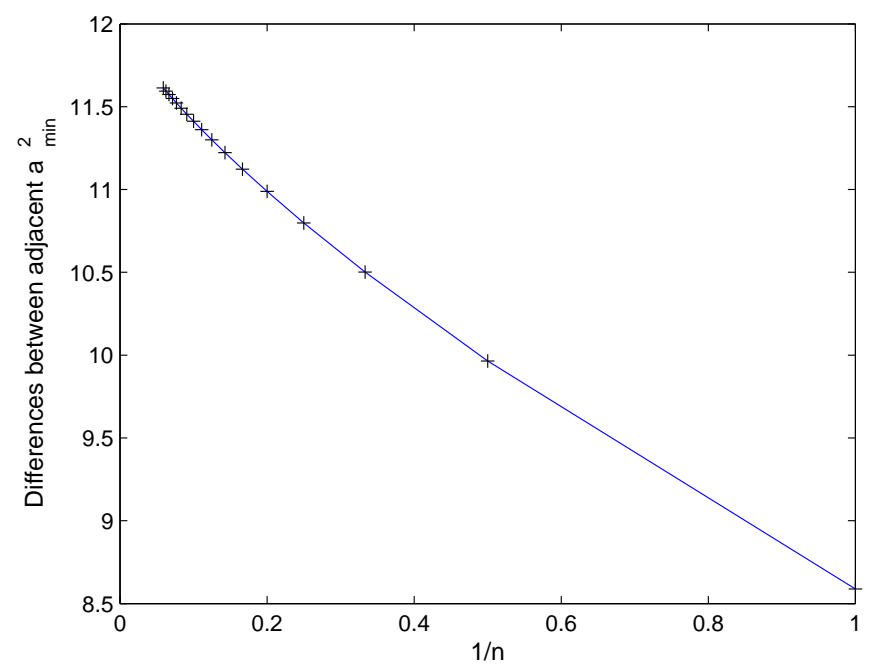

FIG. 1: The differences $\left[a_{\text {crit }}(J+1)\right]^{2}-\left[a_{\text {crit }}(J)\right]^{2}$ taken from Table【plotted as a function of $1 / J$. Observe that as $J$ increases, these differences tend to towards the limiting value 12.

\begin{tabular}{c|c|c}
$J$ & $R_{1}(J)$ series & $R_{1}(J+1)-R_{1}(J)$ \\
\hline 1 & 11.3406567704 & 0.23698190 \\
2 & 11.5776386705 & 0.11060268 \\
3 & 11.6882413518 & 0.06266212 \\
4 & 11.7509034718 & 0.04036140 \\
5 & 11.7912648657 & 0.02824466 \\
6 & 11.8195095305 & 0.02096272 \\
7 & 11.8404722516 & 0.01607921 \\
8 & 11.8565514577 & 0.01300320 \\
9 & 11.8695546582 & 0.01051835 \\
10 & 11.8800730055 & 0.00880552 \\
11 & 11.8888785336 & 0.00746942 \\
12 & 11.8963479526 & 0.00642376 \\
13 & 11.9027717144 & 0.00558923 \\
14 & 11.9083609386 & 0.00491195 \\
15 & 11.9132728866 & 0.00435430 \\
16 & 11.9176271918 &
\end{tabular}

TABLE II: First Richardson extrapolants $R_{1}(J)$ of the sequence of differences $\left[a_{\text {crit }}(J+1)\right]^{2}-$ $\left[a_{\text {crit }}(J)\right]^{2}$ taken from Table I. Notice that $R_{1}(J)$ rises slowly and smoothly towards its limiting value 12 . The differences between successive Richardson extrapolants are also listed.

For the potential $V(x)=x^{6}+2 a x^{4}+\left(a^{2}+4 J-1\right) x^{2}$, the leading-order WKB quantization condition, valid for large $n$, is

$$
\left(2 n+\frac{1}{2}\right) \pi \sim \int_{T_{1}}^{T_{2}} d x \sqrt{E_{n}-V(x)} \quad(n \rightarrow \infty),
$$

where $T_{1,2}$ are the turning points. Note that there is a factor of $2 n+\frac{1}{2}$, rather than $n+\frac{1}{2}$, on 


\begin{tabular}{r|c|c|c|c}
$\mathrm{J}$ & $R_{1}(J)$ & $\mathrm{R}$ of $R_{1}(J)$ & $\mathrm{R}$ of $\mathrm{R}$ of $R_{1}(J)$ & $\mathrm{R}$ of $\mathrm{R}$ of $\mathrm{R}$ of $R_{1}(J)$ \\
\hline 1 & 11.3406567704 & 11.8146205706 & 12.0042728584 & 11.9912792745 \\
2 & 11.5776386705 & 11.9094467145 & 11.9977760665 & 11.9869646719 \\
3 & 11.6882413518 & 11.9388898318 & 11.9941722683 & 11.9887732331 \\
4 & 11.7509034718 & 11.9527104409 & 11.9928225095 & 11.9978459499 \\
5 & 11.7912648657 & 11.9607328547 & 11.9938271976 & 11.9483630075 \\
6 & 11.8195095305 & 11.9662485785 & 11.9862498326 & 12.1168065542 \\
7 & 11.8404722516 & 11.9691059005 & 12.0049007928 & 11.8377031045 \\
8 & 11.8565514577 & 11.9735802620 & 11.9840010818 & 12.0982460411 \\
9 & 11.8695546582 & 11.9747381309 & 11.9966949661 & 11.9726356846 \\
10 & 11.8800730055 & 11.9769338144 & 11.9942890380 & 11.9983156753 \\
11 & 11.8888785336 & 11.9785115620 & 11.9946550959 & \\
12 & 11.8963479526 & 11.9798568565 & & \\
13 & 11.9027717144 & \multicolumn{3}{|l}{}
\end{tabular}

TABLE III: Repeated Richardson extrapolants of the sequence of Richardson extrapolants in Table III. This table provides strong and convincing numerical evidence that Richardson extrapolants $R_{1}(J)$ tend to the limiting value 12 as $J \rightarrow \infty$. This implies that for large $J$ the critical values of $a^{2}$ grow linearly with $J$. See Eq. (13).

the left side of this asymptotic relation because we are counting even-parity eigenfunctions.

For large $n=J$ we approximate the integral in (14) by making the asymptotic substitution $a \sim \sqrt{J} b$, where $b$ is a number to be determined. In order to verify the asymptotic behavior in (13), we must show that $b=\sqrt{12}$. We then make the scaling substitutions

$$
x=y J^{1 / 4} \text { and } \quad E_{J} \sim F J^{3 / 2}
$$

because for large $J$ we can then completely eliminate all dependence on $J$ from the integral. We thus obtain the condition

$$
2 \pi=\int_{y=U_{1}}^{U_{2}} d y \sqrt{F-\left[y^{6}+2 b y^{4}+\left(b^{2}+4\right) y^{2}\right]},
$$

where $U_{1,2}=T_{1,2} J^{-1 / 4}$ are zeros of the algebraic equation

$$
y^{6}+2 b y^{4}+\left(b^{2}+4\right) y^{2}-F=0 .
$$

Next, following the analysis in Ref. [7], we assume that in this large- $J$ limit the polynomial in (17) factors:

$$
\left(y^{2}-\alpha\right)^{2}\left(y^{2}-\beta\right)=0 .
$$

The correctness of this factorization assumption will be verified in the subsequent analysis. We then expand (18);

$$
y^{6}-y^{4}(\beta+2 \alpha)+y^{2}\left(\alpha^{2}+2 \alpha \beta\right)-\alpha^{2} \beta=0 .
$$

Comparing coefficients of like powers of $y$ in (17) and (19), we obtain the three equations

$$
F=\alpha^{2} \beta,
$$




$$
\begin{gathered}
2 b=-2 \alpha-\beta, \\
b^{2}+4=\alpha^{2}+2 \alpha \beta .
\end{gathered}
$$

Subtracting the square of Eq. (21) from three times (22), we get $\beta-\alpha= \pm \sqrt{b^{2}-12}$, and solving this equation simultaneously with (21), we get expressions for $\alpha$ and $\beta$ :

$$
\begin{aligned}
& 3 \alpha=-2 b-\sqrt{b^{2}-12}, \\
& 3 \beta=-2 b+2 \sqrt{b^{2}-12} .
\end{aligned}
$$

We then substitute (23) and (24) into (20) to obtain

$$
F=-\frac{2}{27}\left(b-\sqrt{b^{2}-12}\right)\left(2 b+\sqrt{b^{2}-12}\right)^{2} .
$$

Finally, we calculate the value of the number $b$. Our procedure is simply to show that the special choice $b^{2}=12$ is consistent with the limiting WKB integral in (16). With this choice we can see from (23) and (24) that $\alpha=\beta=4 / \sqrt{3}$ and that (16) reduces to

$$
2 \pi=\int_{y=-\alpha}^{\alpha} d y\left(\alpha-y^{2}\right)^{3 / 2}=2 \int_{y=0}^{\alpha} d y\left(\alpha-y^{2}\right)^{3 / 2}
$$

We simplify this integral by making the substitution $y=\sqrt{u \alpha}$, and obtain

$$
\frac{3}{8} \pi=\int_{u=0}^{1} d u u^{-1 / 2}(1-u)^{3 / 2}
$$

which is an exact identity. Thus, we may conclude that $b^{2}=12$. This verifies the asymptotic formula in (13) for the location of the $\mathcal{P} \mathcal{T}$ boundary.

Furthermore, we can see that $F=\frac{64}{9} \sqrt{3} \approx 12.3$. Thus, we obtain a formula for the large- $J$ asymptotic behavior of the largest QES eigenvalue at the $\mathcal{P} \mathcal{T}$ boundary:

$$
E_{J} \sim \frac{64}{9} \sqrt{3} J^{3 / 2} \quad(J \rightarrow \infty)
$$

The difference between this WKB calculation and that done in Ref. [7] for the Hermitian QES sextic Hamiltonian (11) is that here we have a critical value, $b=\sqrt{12}$, or $a \sim \sqrt{12 J}$. This critical value defines the boundary between the regions of broken and unbroken $\mathcal{P} \mathcal{T}$ symmetry for the $\mathcal{P} \mathcal{T}$-symmetric Hamiltonian in (17). There is no analog of this boundary for Hermitian Hamiltonians.

\section{Acknowledgments}

We are greatful to Dr. H. F. Jones for giving us valuable advice with regard to our WKB approximations. CMB is grateful to the Theoretical Physics Group at Imperial College for its hospitality and he thanks the U.K. Engineering and Physical Sciences Research Council, the John Simon Guggenheim Foundation, and the U.S. Department of Energy for financial support. MM gratefully acknowledges the financial support of ???. 
[1] See A. G. Ushveridze, Quasi-Exactly Solvable Models in Quantum Mechanics (Institute of Physics, Bristol, 1993) and references therein.

[2] A. V. Turbiner, Sov. Phys., JETP 67, 230 (1988), Contemp. Math. 160, 263 (1994), and M. A. Shifman, Contemp. Math. 160, 237 (1994).

[3] A. V. Turbiner, Comm. Math. Phys. 118, 467 (1988).

[4] M. A. Shifman and A. V. Turbiner, Comm. Math. Phys. 126, 347 (1989).

[5] A. González-López, N. Kamran, and P. J. Olver, Comm. Math. Phys. 153, 117 (1993) and Contemp. Math. 160, 113 (1994).

[6] C. M. Bender and G. V. Dunne, J. Math. Phys. 37, 6 (1996).

[7] C. M. Bender, G. V. Dunne, and M. Moshe, Phys. Rev. A 55, 2625 (1997).

[8] C. M. Bender and S. Boettcher, Phys. Rev. Lett. 80, 5243-5246 (1998).

[9] C. M. Bender, S. Boettcher, and P. N. Meisinger, J. Math. Phys. 40, 2201-2229 (1999).

[10] P. Dorey, C. Dunning and R. Tateo, J. Phys. A 34 L391 (2001); ibid. 34, 5679 (2001).

[11] G. Lévai and M. Znojil, J. Phys. A33, 7165 (2000); B. Bagchi and C. Quesne, Phys. Lett. A300, 18 (2002); Z. Ahmed, Phys. Lett. A294, 287 (2002); G. S. Japaridze, J. Phys. A35, 1709 (2002); A. Mostafazadeh, J. Math. Phys. 43, 205 (2002); ibid; 43, 2814 (2002); D. T. Trinh, PhD Thesis, University of Nice-Sophia Antipolis (2002), and references therein.

[12] An excellent summary of the current status and the background of non-Hermitian and $\mathcal{P} \mathcal{T}$-symmetric Hamiltonians may be found in F. Kleefeld, hep-th/0408028 and hep-th/0408097.

[13] C. M. Bender, D. C. Brody, and H. F. Jones, Phys. Rev. Lett. 89, 270401 (2002) and Am. J. Phys. 71, 1095 (2003).

[14] C. M. Bender and A. Turbiner, Phys. Lett. A 173, 442 (1993).

[15] C. M. Bender and S. Boettcher, J. Phys. A: Math. Gen. 31, L273 (1998).

[16] For a nonpolynomial QES $\mathcal{P} \mathcal{T}$-symmetric Hamiltonian see A. Khare and B. P. Mandal, Phys. Lett. A 272, 53 (2000).

[17] Notice that $\psi(x)$ in (8) is a function of $x^{2}$ and thus all of the QES wave functions are symmetric under parity reflection $(x \rightarrow-x)$. In general, $\mathcal{P} \mathcal{T}$-symmetric Hamiltonians, such as $H=p^{2}-x^{4}$ are not symmetric under parity reflection because the parity operator $\mathcal{P}$ changes the complex domain of the Hamiltonian operator. As a consequence, the expectation value of the $x$ operator is nonvanishing. [See C. M. Bender, P. N. Meisinger, and H. Yang, Phys. Rev. D 63, 45001 (2001).] Nevertheless, the special QES eigenfunctions in (8) are parity-symmetric. We believe that the parity operator may therefore be used to distinguish between the QES and the non-QES portions of the Hilbert space. 
[18] C. M. Bender and S. A. Orszag, Advanced Mathematical Methods for Scientists and Engineers, (McGraw-Hill, New York, 1978), Chap. 10. 